\title{
LA TUTORÍA EN PROGRAMAS DE DOCTORADO. TENSIONES TUTORIALES DOCTORADO EN CIENCIAS DE LA EDUCACIÓN RUDECOLOMBIA $^{1}$
}

\author{
Mario Gabriel Torres Calixto ${ }^{2}$ \\ Universidad Pedagógica y Tecnológica de Colombia - Colombia \\ Grupo de investigación HISULA \\ magatoca@yahoo.com
}

Recepción: 10/04/2011

Evaluación: 09/05/2011

Aceptación: 9/11/2011

Artículo de Reflexión

\section{RESUMEN}

El objeto del trabajo es el de analizar las tensiones existentes en el desarrollo de la tutoría del Doctorado en Ciencias de la Educación de RUDECOLOMBIA, dentro del contexto de las tutorías internacionales, con la pregunta ¿Inciden las tensiones de la tutoría en la calidad del trabajo y formación integral del doctorando?.

El método se centra desde la historia de la educación que permitió establecer el modelo para analizar el objeto de estudio como es la tutoría en un programa de doctorado. La metodología cualitativa junto con la comparativa, nos proporcionó los procedimientos, clasificación y organización de la información, que permitieron comprender el lugar que ha ocupado la tutoría en la formación de los doctores en RUDECOLOMBIA, desde la revisión de literatura nacional e internacional, las contextualiza dentro del concepto de globalización y plantea alternativas de solución. Las estrategias se centraron

1 El trabajo presenta los avances de la tesis doctoral del autor, titulada "La tutoría en la formación de doctores en educación de RUDECOLOMBIA. 1998 - 2008”, que se realiza en el Doctorado Ciencias de la Educación de RUDECOLOMBIA bajo la dirección de la Dra. Diana Soto Arango.

2 Candidato a doctor del Doctorado en Ciencias de la Educación de RUDECOLOMBIA CADE - UPTC, integrante del grupo de investigación Historia y Prospectiva de la Universidad Latinoamericana clasificado A1 COLCIENCIAS de la Universidad Pedagógica y Tecnológica de Colombia. 
en la información de los talleres realizados en Comité Curricular y en las 61 entrevistas realizadas y el archivo de RUDECOLOMBIA. Los resultados permitieron identificar, que si bien existen normas sobre la tutoría en los doctorados, aún se identifican situaciones en las cuales, la decisión a tomar, depende de la autonomía del tutor. Esto genera tensiones, puesto que pueden existir argumentos a favor o en contra de dicha decisión, lo cual incide en la calidad de la formación doctoral.

Se socializan algunas recomendaciones para implementar la normatividad y facilitar la toma de decisiones en relación con los dilemas y tensiones que surjan en el proceso de formación del graduado.

Palabras clave: Revista Historia de la Educación Latinoamericana, tutoría, tensiones, formación doctoral, doctorado RUDECOLOMBIA, formación de tutores.

\title{
THE TUTORSHIP IN PROGRAMS OF DOCTORATE TUTORIAL TENSIONS AT THE DOCTORAL PROGRAM IN EDUCATIONAL SCIENCES OF RUDECOLOMBIA
}

\author{
Mario Gabriel Torres Calixto \\ Universidad Pedagógica y Tecnológica de Colombia - Colombia \\ Research group HISULA \\ magatoca@yahoo.com
}

\begin{abstract}
The object of the work is to analyze the existing tensions in the development of the mentoring of the Doctoral Program in Educational Sciences of RUDECOLOMBIA, within the context of the international mentoring, with the question: ¿Do the tensions of the mentoring affect the quality of the work and integral formation of the doctoral student?

The method focuses on the history of education that led to setting up the model to analyze the object of study as is the mentoring in a doctorate program. The qualitative methodology along with the comparative, provided us with the procedures, classification and organization of information that allowed us to understand the place that has occupied the mentoring in the training of doctors in RUDECOLOMBIA, from a review of national and international literature, they are contextualized within the concept of globalization and
\end{abstract}


poses alternatives of solution. The strategies focused on the information about the workshops performed at the Curriculum Committee, in the 61 interviews carried out and the revision of the files of RUDECOLOMBIA. The results allowed us to identify that although there are rules on mentoring in the doctorate, there are still situations in which, the decision to take, depends on the autonomy of the mentor. This creates tensions, since there can be arguments in favor of or against such a decision, which affects the quality of the doctoral training.

Conclusions: some recommendations are presented to implement the regulations and facilitate the decision-making in relation with the dilemmas and tensions that arise in the process of formation of the graduate; in addition, a proposal for training and/or permanent seminar of update for the mentor is presented.

Key words: Journal of Latin American Education History, tutorship, doctoral training, tensions, tutor training.

\title{
A TUTORIA EM PROGRAMAS DE DOUTORADO. TENSÕES TUTORIAIS NO DOUTORADO EM CIÊNCIAS DA EDUCAÇÃO RUDECOLOMBIA
}

\author{
Mario Gabriel Torres Calixto \\ Universidad Pedagógica y Tecnológica de Colombia - Colombia \\ Grupo de pesquisa HISULA \\ magatoca@yahoo.com
}

\section{RESUMO}

O objetivo deste trabalho é analisar as tensões existentes no desenvolvimento da tutoria do Doutorado em Ciências da Educação de RUDECOLOMBIA, dentro do contexto das tutorias internacionais, com a pergunta: incidem as tensões da tutoria na qualidade do trabalho e na formação integral do doutorando?.

O método é centrado desde a história da educação que permitiu estabelecer o modelo para analisar o objeto de estudo como é a tutoria em um programa de doutorado. A metodologia qualitativa junto com a comparativa nos proporcionou os procedimentos, classificação e organização da informação, que permitiram compreender o lugar que ocupou a tutoria na formação dos doutores em RUDECOLOMBIA, desde 
a revisão de literatura nacional e internacional, as contextualiza dentro do conceito de globalização e oferece alternativas de solução. As estratégias se centraram na informação das oficinas realizadas no Comitê Curricular, nas 61 entrevistas realizadas e no arquivo de RUDECOLOMBIA. Os resultados permitiram identificar que, ainda que existam normas sobre a tutoria nos doutorados, ainda se identificam situações nas quais a decisão a ser tomada depende da autonomia do tutor. Isto gera tensões, posto que podem existir argumentos a favor ou contra a dita decisão, o que incide na qualidade da formação doutoral.

Socializam-se algumas recomendações para implementar a normatividade e facilitar a tomada de decisões em relação com os dilemas e tensões que surjam no processo de formação do graduado.

Palavras-chave: Revista História da Educação Latino-americana, tutoria, tensões, formação doutoral, doutorado RUDECOLOMBIA, formação de tutores.

\section{INTRODUCCIÓN}

El trabajo hace parte de la tesis doctoral de "La tutoría en la formación de doctores en educación de RUDECOLOMBIA, 1998-2011”. Inicialmente se presenta un análisis internacional de los problemas generales del doctorado y los específicos de la actividad tutorial, junto con las alternativas de solución; más adelante se plantea el cómo debe ser la tutoría para luego describir el concepto de tensión. Se analizan algunas tensiones relacionadas con la tutoría, diagnosticadas durante el Seminario Taller sobre Tutoría y Dirección de Tesis, realizado por el Comité Curricular Nacional y de Autoevaluación y se proponen alternativas de solución; posteriormente se mencionan otras tensiones que pueden surgir en la actividad tutorial y finalmente se menciona el contexto, dentro de la sociedad del conocimiento, que está afectando el desarrollo de la tutoría y la formación doctoral. Al final se presenta una propuesta de programa de formación tutorial, aplicable al Doctorado en Ciencias de la Educación de RUDECOLOMBIA desde los planteamientos del grupo en el cual se ubica la investigación: "Historia y prospectiva de la Universidad Latinoamericana", HISULA.

Consideramos que la tutoría es central en el proceso de formación doctoral, porque su objetivo es la formación integral del graduado en los aspectos físico, mental, social, ético, profesional e investigativo. Esto hace que puedan surgir diferentes situaciones que originen tensiones, las cuales afectan la calidad del graduado. 
Dado que la reglamentación actual no es clara en relación con las responsabilidades y funciones, tanto de tutores, como de estudiantes, se hace necesario que en el doctorado se analicen las diferentes situaciones que pueden surgir en el desarrollo de la actividad tutorial, incluyendo las tensiones, con el objeto de contextualizar la normatividad. Esta ponencia es una contribución al trabajo que se ha venido desarrollando en el doctorado, en búsqueda del mejoramiento de la calidad del programa.

En relación con la prospectiva de la tutoría, esta actividad se contextualiza desde la sociedad del conocimiento y se plantean las tendencias en la formación doctoral.

Teniendo en cuenta la diversidad de denominaciones existentes en los diferentes países, que tiene la actividad de acompañamiento y asesoría al estudiante durante su formación doctoral, se sugiere que dentro de la normativa a establecer sobre dicha actividad, se deje uno de los dos nombres actuales, tutor o director de tesis, con el objeto de evitar confusiones y establecer específicamente sus características, funciones y responsabilidades.

Respecto al método se centra desde la historia de la educación que permitió establecer el modelo para analizar el objeto de estudio como es la tutoría en un programa de doctorado. La metodología cualitativa junto con la comparativa nos proporcionó los procedimientos, clasificación y organización de la información que permitieron comprender el lugar que ha ocupado la tutoría en la formación de los doctores en RUDECOLOMBIA desde la revisión de literatura nacional e internacional, las contextualiza dentro del concepto de globalización y plantea alternativas de solución. Las estrategias se centraron en la información de los talleres realizados en Comité Curricular y en las 61 entrevistas realizadas y el archivo de RUDECOLOMBIA. Los resultados permitieron identificar que si bien existen normas sobre la tutoría en los doctorados, aún se identifican situaciones en las cuales, la decisión a tomar, depende de la autonomía del tutor. Esto genera tensiones, ya que pueden existir argumentos a favor o en contra de dicha decisión, lo cual incide en la calidad de la formación doctoral. En las conclusiones se socializan algunas recomendaciones para implementar la normatividad y facilitar la toma de decisiones en relación con los dilemas y tensiones que surjan en el proceso de formación del graduado.

\section{Análisis internacional de los problemas del doctorado}

En primera instancia analizamos algunas de las tensiones de los doctorados, en general, en el Reino Unido, Alemania, Estados Unidos, Australia, México y Brasil, con el propósito de presentar una mirada amplia de la formación doctoral. Más adelante se mencionan algunas de las 
alternativas de solución a la problemática planteada. La razón por la cual se revisan estos países, es que constituyen una muestra representativa de la actividad de formación doctoral a nivel mundial.

Es así que en el Reino Unido, Green ${ }^{3}$ plantea problemas relacionados con la pérdida del nivel internacional, la disminución del logro de competencias, tiempo de terminación prolongado y bajas tasas de finalización. Por su parte, en Alemania, $\mathrm{Kehm}^{4}$ menciona insuficiente estructura del programa, aumento del tiempo de terminación, alta deserción, alta dependencia del tutor, falta de enfoque interdisciplinar e insuficiente orientación al mercado laboral. Asimismo, hemos localizado que en Australia, Kemp ${ }^{5}$ identifica poca concentración en áreas de fortaleza investigativa, falta de preparación laboral, pérdida de recursos públicos y privados asociados a tiempos prolongados de finalización y bajo porcentaje de terminación; además poca relación de la investigación con sistemas de innovación y escaso apoyo financiero a programas de diversificación y excelencia.

En cuanto a Estados Unidos, Nerad ${ }^{6}$ detecta que la formación docente es inadecuada, el tiempo de terminación prolongado, las tasas de finalización bajas, la preparación laboral deficiente y las competencias para trabajar en equipo insuficientes. Otro análisis localizamos en Brasil, a través de Ribeiro ${ }^{7}$ quien manifiesta que no existe articulación con el pregrado, la distribución geográfica es inequitativa, hay proliferación de programas de ciencias sociales aplicadas y dificultad para el mantenimiento de programas que contribuyen al desarrollo social y económico del país, tales como ciencias básicas, ingeniería y tecnología ${ }^{8}$. Otro problema que se analiza desde México, con

3 GREEN, Howard. (2008): Doctoral Education in Europe. United Kingdom, en Toward a global PhD: Forces and forms in doctoral education worldwide. NERAD, Maresi y HEGGELUND, Mimi. Seattle. Washington University Press. pp. 36-74.

4 KEHM, Bárbara. (2008): Doctoral Education in Europe. Germany, en Toward a global PhD: Forces and forms in doctoral education worldwide. NERAD, Maresi. y HEGGELUND, Mimi. Seattle. Washington University Press. pp. 19-35.

5 KEMP, David. (1999): Knowledge and innovation: a policy statement and research training. Camberre, Departament of Education, Training and Youth Affairs.

6 NERAD, Maresi. (2008): Doctoral Education in North America. United States of America, en Toward a global PhD: Forces and forms in doctoral education worldwide. NERAD, Maresi y HEGGELUND, Mimi. Seattle. Washington University Press, pp. 278-299.

RIBEIRO, Renato. (2008): Doctoral Education in África, South América, and México. Brazil, en Toward a global PhD: Forces and forms in doctoral education worldwide. NERAD, Maresi y HEGGELUND, Mimi. Seattle. Washington University Press, pp. 131-145.

8 RIBEIRO, Renato. (2007): Doctoral education in Brazil, en The doctorate worldwide. POWELL, Stuart y GREEN, Howard. Maidenhead. MacGrawHill, pp. 143-154. 
Alcántara, Malo y Fortes ${ }^{9}$ mencionan la alta concentración de los graduados en los grandes centros urbanos, la poca influencia en el crecimiento de producción del país, el bajo factor de impacto de las publicaciones en relación con el promedio mundial, la falta de articulación con el pregrado, la deficiente inversión del sector productivo en programas de investigación, la falta de correlación del programa de formación doctoral con los propósitos de desarrollo investigativo del país, la tendencia a producir conocimiento utilizable solo en la academia, la inadecuada preparación laboral y la baja producción investigativa.

En definitiva, podemos decir que se establecen las tendencias de la correlación de competencias con el tiempo en la finalización de la tesis, la incidencia de la estructura del programa en el tiempo y deserción, la preparación laboral del graduado y la exigencia cada vez mayor a la formación y/o actualización del tutor.

En estos países las alternativas de solución se centran en el Reino Unido $^{10}$ donde se ha establecido desde el año 1998, actualizado a 2004, un "código de práctica", respaldado por numerosas organizaciones y autoridades, el cual plantea, para el posgrado, principios generales que garantizan el aseguramiento de su calidad ${ }^{11}$. Otras decisiones han sido otorgar auxilios económicos a programas de alta calidad, dar mayor énfasis a la formación investigativa antes de iniciar el doctorado, estimular la organización del doctorado con administración autónoma y establecer mayor énfasis a la tutoría y formación de tutores ${ }^{12}$.

En Alemania, se implementó el proceso de selección y admisión, el establecimiento de deberes y derechos del tutor y el estudiante, se estableció la tutoría en equipo, el desarrollo de cursos académicos, la participación en proyectos de investigación interdisciplinaria y el desarrollo de la tutoría, respaldado por la elaboración de un contrato entre las partes ${ }^{13}$.

9 ALCÁNTARA, Armando, MALO, Salvador y FORTES, Mauricio. (2008): Doctoral Education in Africa, South America, and México. México, en Toward a global PhD: Forces and forms in doctoral education worldwide. NERAD, Maresi y HEGGELUND, Mimi. Seattle. Washington University Press, pp. 146-170. MALO, Salvador. (2007): Doctoral education in México, en The doctorate worldwide. POWELL, Stuart. y GREEN, Howard. Maidenhead. MacGraw-Hill, pp. 194-204.

${ }^{10}$ GREEN, Howard. (2008): Op., cit, p. 36.

${ }^{11}$ QUALITY ASSURANCE AGENCY (QAA). (2004): Code of practice for the Assurance of Academic Quality and Standards in Higher Education: Section 1: Posgraduate research, (2nd Ed). Gloucester, Quality Assurance Agency

12 GREEN, Howard. (2008): Óp., cit, p. 60.

${ }^{13}$ KEHM, Barbara. (2008): Óp., cit, p. 25. 
En Australia, el Consejo Australiano de Decanos y Directores de Estudios de Posgrado"14, plantea que "el tutor principal tiene la responsabilidad de proporcionar y coordinar el apoyo y la asesoría para la candidatura". Este tutor debe tener, aparte de los requisitos tradicionales, "entrenamiento y/o experiencia en el proceso de tutoría".

Por otra parte, en Estados Unidos, se ha establecido la importancia que tienen la monitoría, el estudio y la evaluación del impacto de los programas, la necesidad de responder a las fuerzas externas del mercado, el llamado a la reconciliación de las fuerzas internas institucionales que obstaculicen la asignación presupuestal y la promoción del personal académico; de igual manera se estimula la innovación en investigaciones que den respuesta a necesidades de la sociedad y contribuyan a la creación de conocimiento. ${ }^{15}$

En resumen, existen problemáticas comunes en todos los países analizados, como los tiempos de terminación del programa, las tasas de finalización, poca relación con el sector productivo, la no preparación del estudiante para el empleo y la tendencia de los programas de graduar doctores para que trabajen específicamente en universidades, entre otras.

También se evidencian tendencias a nivel mundial como el aseguramiento de la calidad, el crecimiento del número de doctorados, la diversificación de los admitidos a los doctorados, la internacionalización, doble titulación, entre otras, que inciden en la formación doctoral y en las exigencias cada vez mayores y complejas hacia las competencias que debe tener el tutor. De igual manera se anotan alternativas de solución que algunos países están implementando, para dar respuesta a la problemática identificada.

En la siguiente sección se plantean los problemas del doctorado en los que influye la práctica tutorial.

\section{Dificultades y problemas del doctorado relacionados con la actividad tutorial}

En este sentido, el Consejo de Escuelas de Posgrado de los Estados Unidos, en un proyecto donde participaron 29 universidades, encontró que la terminación en el tiempo previsto y las tasas de finalización estaban

${ }^{14}$ COUNCIL OF AUSTRALIAN DEANS AND DIRECTORS OF GRADUATE STUDIES. (2008): Framework for Best Practice in Doctoral Research Education in Australia. Townsville, Australia, DDOGS. Descargado el 23 de julio de 2011, de http:/ /www.ddogs.edu.au/files?folder_ id $=2123770849$

${ }^{15}$ NERAD, Maresi. (2007): "Doctoral education in USA", en: The doctorate worldwide. POWELL, Stuart. y GREEN, Howard. (2008): Maidenhead. MacGraw-Hill, pp. 133-140. 
relacionadas con la tutoría efectiva ${ }^{16}$. Es así que el Departamento de Educación, Entrenamiento y Asuntos de la Juventud de Australia ${ }^{17}$, encontró que la falta de acuerdos sobre la actividad tutorial, junto con factores ambientales institucionales y las características propias de las cohortes, influyeron directamente en la tasa de finalización del doctorado.

Es necesario recordar que dentro de las competencias que debe lograr el estudiante doctoral, con la orientación del tutor, están las relacionadas con el desarrollo profesional y preparación para su carrera doctoral ${ }^{18}$, incluyendo el aspecto laboral. Es relevante indicar que en un estudio realizado en el Reino Unido ${ }^{19}$, con participación de 172 tutores, el 77\% planteó no tener experiencia de trabajo con guías de tutoría, aunque las consideraban útiles. El 20\% opinó que la formación, aparte de la investigación académica, debiera incluir competencias en administración, industria y gobierno. Asimismo, el estudio de Clements y Alcántara ${ }^{20}$, realizado en tres instituciones de investigación en posgrado de USA y México, mostró que la eficiencia de la tutoría y la obtención del grado en el tiempo esperado, están relacionadas con aspectos como confianza interpersonal, compromiso del estudiante en el proyecto de investigación, compartido con el tutor, líneas claras de investigación, existencia de profesores y estudiantes de tiempo completo y alta responsabilidad del tutor en el progreso académico de los estudiantes. Estos hallazgos confirman la influencia que tiene una tutoría eficaz en la terminación del programa en el tiempo esperado.

Por su parte, hemos localizado que en Alemania, la utilización del modelo maestro-aprendiz, hizo que se creara una alta dependencia del estudiante hacia el tutor ${ }^{21}$. Esta situación generó que algunos tutores utilizaran a los estudiantes para desarrollar sus propias obligaciones investigativas y

${ }^{16}$ COUNCIL OF GRADUATE SCHOOLS. (2004): Completion project. Washington, CGS. Descargado el 23 de julio de 2011, de http://www. phdcompletion.org/promising/mentoring.asp

17 DEPARTMENT OF EDUCATION, TRAINING AND YOUTH AFFAIRS, AUSTRALIA. (2001) "Factors Associated with completion of research higher degrees", en Higher education series Report. No. 37. Sydney, Higher Education Division.

${ }^{18}$ COUNCILOF AUSTRALIAN DEANS AND DIRECTORS OF GRADUATE STUDIES. (1999): Statement on Skills Development for Research Students. Townsville, Australia, DDOGS. Descargado el 23 de julio de 2011, de http:// www.ddogs.edu.au/files?folder_id=2123770849

19 THE WELLCOME TRUST. (2001): Review of Wellcome Trust PhD Training. The supervisor perspective. London, Wellcome Trust.

${ }^{20}$ CLEMENTS, Margaret y ALCÁNTARA Armando. (2005) "Mentoring practices in doctoral program in Mexico and the United States: Growing Wiser Together", paper prepared for the $49^{\text {th }}$ Annual Meeting of the comparative and international Education Society, 22-26 March. Palo Alto, CA, Stanford University.

${ }^{21}$ KEHM, Bárbara. (2008): Óp., cit, p. 20. 
docentes, lo cual aumentaba el tiempo para obtener el grado ${ }^{22}$. Una de las causas de esta problemática fue la falta de conciencia, compromiso personal y preparación del tutor.

En resumen, algunos de los problemas presentados en el desarrollo del doctorado, tales como el prolongado tiempo de terminación, las bajas tasas de finalización, el no desarrollo de competencias diferentes a las académicas y la utilización del estudiante en actividades diferentes a sus responsabilidades, están relacionadas en gran medida con la actividad tutorial. En algunas ocasiones el tutor no tiene conocimiento de las políticas tutoriales, lo cual dificulta más el desarrollo de su práctica.

\section{Alternativas de solución para el aseguramiento de la calidad de la actividad tutorial}

Tal como se ha analizado, algunos de los problemas de la formación doctoral a nivel mundial, están directamente relacionados con la práctica tutorial. Conscientes de esta situación, en algunos países se han presentado alternativas que ayuden a mejorar estas dificultades, mediante la reglamentación de la tutoría y la formación de los tutores, en las competencias necesarias para su desempeño con calidad. Inicialmente se presentan algunas consideraciones acerca de la necesidad de formación tutorial, luego ejemplos de programas estructurados para suplir dicha necesidad y finalmente sitios web con recursos electrónicos.

En el Reino Unido, el Código de Práctica de la Agencia de Aseguramiento de la Calidad, en el precepto $11^{23}$, plantea que todos los tutores necesitan tener capacidades para desempeñar su rol, para lo cual las instituciones deben garantizar actividades de desarrollo continuo y actualización en las competencias necesarias y la socialización de buenas prácticas. Además existen otras publicaciones que han planteado el tema de la formación del tutor, en las cuales se evidencia la importancia dada a la formación tutorial, para mejorar la calidad del doctorado. Por ejemplo, el Consejo Europeo de Candidatos Doctorales y nuevos investigadores ${ }^{24}$, plantea la necesidad que los tutores nuevos reciban entrenamiento de los tutores con mayor experiencia; de igual manera sugiere que los tutores con mayor experiencia participen en cursos de actualización, con el objeto de garantizar que aún se poseen los estándares requeridos.

${ }^{22}$ KEHM, Bárbara. (2007): Doctoral Education in Germany: between tradition and reform, en The doctorate worldwide. POWELL Stuart y GREEN Howard. Maidenhead. McGraw-Hill, pp. 52-63.

${ }^{23}$ QUALITY ASSURANCE AGENCY (QAA). (2004): Óp., cit, p. 14.

${ }^{24}$ EUROPEAN COUNCIL OF DOCTORAL CANDIDATES AND YOUNG RESEARCHERS (EURODOC). (2004): Supervision and training charter for early stage researchers. Brussels, EURODOC. Descargado el 29 de julio de 2011, de http://www.eurodoc.net/temp_policies 
Es así que, Moreno (2007) ${ }^{25}$ sostiene que existe la convicción errada, que quien tiene el título de doctor y es un investigador activo, puede desempeñar el papel de tutor para formar investigadores. En su trabajo demuestra que el desarrollo de la actividad tutorial puede ubicarse en diferentes niveles de calidad. Así mismo, Green planteó que la tutoría y la investigación son dos competencias diferentes y que un buen investigador no es necesariamente un buen tutor. Este planteamiento, complementado con el aumento progresivo de reclamos y demandas de los estudiantes insatisfechos con la tutoría, ha hecho que las universidades tomen más seriamente la calidad de la tutoría y el concepto de formación del tutor ${ }^{26}$.

\section{Programas de formación del tutor}

De los programas más importantes que hemos localizado podemos mencionar los TAPPS: Entrenamiento y Acreditación para Tutores de Postgrado, en el Reino Unido y FIRST: para el Mejoramiento de la Tutoría y la Formación del tutor, en Australia. Además, algunas universidades desarrollan programas de manera autónoma, por ejemplo Oxford, Bradford y Sheffield en el Reino Unido, y Sidney, Nacional y Monash en Australia.

Es así que el programa TAPPS que se estableció en 1997 en el Reino Unido, presenta como objetivo dar entrenamiento y desarrollo de competencias generales y específicas de la tutoría. Es el primer programa acreditado por la Academia de Educación Superior, la cual permite que se otorgue el título profesional de tutor. El programa capacita al tutor para que forme integralmente al estudiante, le garantice la realización de la investigación y la presentación exitosa de la tesis ${ }^{27}$.

Por su parte, FIRST que se organizó por 45 universidades australianas, de Nueva Zelanda y Europeas, presentó como objetivo central el logro de competencias que son deseables en la tutoría, particularmente en aquellos de quienes se espera que trabajen efectivamente en los nuevos ambientes de investigación. Además, proporciona acceso a una gama de recursos que

${ }^{25}$ MORENO, María. (2007) "Experiencias de formación y formadores en programas de doctorado en educación", en: Revista Mexicana de Investigación Educativa. Vol. 12. No. 33. Guadalajara, Universidad de Guadalajara, pp. 561-580.

${ }^{26}$ URQUHART, Kirstie. (2001) "Superior supervisión", en: Science Career Magazine. noviembre 02 de 2001. Nueva York, American Association for the Advancement of Science. Descargado el 23 de julio de 2011, de: http://sciencecareers.sciencemag.org/career_magazine/previous_issues/ articles/2001_11_02/noDOI.5857770442054790723

${ }^{27}$ THE HIGHER EDUCATION ACADEMY (HEA).( 2005): First accredited programme for training in supervising postgrads. Heslington, HEA. Descargado el 24 de julio de 2011, de: http://www.heacademy.ac.uk/news/ detail/accredited_programme_training_supervising_postgrads 
ayudan a las universidades y a los tutores individuales a mejorar la calidad de la educación doctoral ${ }^{28}$.

Debemos indicar que, las páginas web con recursos electrónicos, se puede consultar documentos, producto de la experiencia en formación del tutor, los cuales orientan los programas de entrenamiento. Entre éstas, podemos destacar las siguientes: Vitae, Pat Cryer-recursos para tutores y MORE: tutoría y otros recursos para la excelencia. Además, es relevante la Vitae que es una organización patrocinada por el Consejo de Investigación del Reino Unido (RCUK) y manejada por la Organización de Desarrollo Profesional y Disciplinar (CRAC). Esta organización, lidera el desarrollo personal, profesional y disciplinar de investigadores doctorales y del personal relacionado con investigación. Persigue el logro de competencias de alto nivel e innovación, para producir investigadores de clase mundial. Una de las cuatro áreas de trabajo es la relacionada con la formación de tutores y administradores. Dentro de los principales recursos están el Boletín Overview y el centro de recursos para los tutores del doctorado ${ }^{29}$.

Sin lugar a dudas el Pat Cryer-Recursos para supervisores, presenta sugerencias, consejos, conceptos clave, soporte general para estudiantes del doctorado, tutores, profesores, administradores y personal en entrenamiento. Plantea un marco de referencia para el desarrollo de programas de entrenamiento para el tutor. Esta página fue creada por la Doctora Pat Cryer, docente de las universidades de Manchester y de Winchester, del Reino Unido ${ }^{30}$.

Por su parte el MORE, ofrece guías para el tutor, seminarios talleres para la formación tutorial, enlaces de interés, recursos bibliográficos por temas y descarga de documentos sobre la actividad tutorial. Está patrocinada por la Escuela de Graduados de Rackham de la Universidad de Michigan ${ }^{31}$.

${ }^{28}$ FOR IMPROVING RESEARCH SUPERVISION AND TRAINING CONSORTIUM (fIRST). (2004): How FIRST Works Melbourne, Australia, FIRST. Descargado el 25 de Julio de 2011, de http://www.first.edu.au/index. html

${ }^{29}$ VITAE. (s.f.): Supervisors $\mathcal{E}$ managers. Cambridge, Careers Research and Advisory Centre (CRAC). Descargado el 25 de julio de 2011, de: http://www. vitae.ac.uk/policy-practice/1389/Supervisors--managers.html.

${ }^{30}$ CRYER, Pat. (2006): Educational resources for postgraduate research. Manchester, University of Manchester. Descargado el 23 de julio de 2011, de http://www.postgrad_resources.btinternet.co.uk/index.htm

${ }^{31}$ MENTORING OTHERS RESULTS IN EXCELLENCE (MORE). (2011): Graduate student mentoring at the University of Michigan. Michigan, University of Michigan. Descargado el 29 de julio de 2011, de http://www. more.umich.edu/mentoring/graduate-student-mentoring/. 
En resumen, esta revisión presenta a los diferentes países dentro de las políticas en los contextos gubernamental e institucional. Es importante señalar que estas políticas tienen en cuenta la complejidad de las actividades que tiene que desarrollar el tutor y por lo tanto consideran necesario que existan programas que garanticen su formación y actualización. Pero, además de los programas formales, se presenta interés en que se proporcionen los recursos necesarios, ya sea para que participen en cursos o que desarrollen actividades de autoformación, utilizando herramientas electrónicas. Aunque no todas las universidades están en el proceso, es cada vez mayor el interés, el establecimiento de políticas y la asignación de recursos para que la formación del tutor sea uno de los principales objetivos de los programas de doctorado.

\section{Tensiones relacionadas con la tutoría}

Concebimos al tutor dentro de la concepción de la Dra. Soto que indica que "en un programa doctoral es un doctor que se involucra en el proceso de formación integral de un doctorando en la construcción de una tesis" 32 . Por otra parte, entendemos la tutoría como el proceso en el cual un investigador experimentado, con características personales, pedagógicas y académicas específicas acompaña y orienta a un estudiante de doctorado, con el objeto de apoyar su formación integral como investigador autónomo y elaborar una tesis doctoral de alto impacto. Otros autores presentan esta actividad como el acompañamiento del estudiante en su proceso de aprendizaje y formación en investigación, para convertirlo en un investigador autónomo e innovador, acreditado en su disciplina ${ }^{33}$. Por su parte, la Dra. Soto señala que "la tutoría consiste en la mediación investigativa entre un doctor y un doctorando en la elaboración de una tesis donde se construye nuevo conocimiento desde la co-responsabilidad, la autonomía intelectual mediada por la flexibilidad curricular de la ruta académica que incluye la experiencia investigativa de redes académicas internacionales ${ }^{34}$.

Dentro de las características de una tutoría efectiva están las de flexibilizar el proceso a medida que la investigación avanza y el estudiante se desarrolla, socializar con los estudiantes lo que ellos consideran que es una buena tutoría, tener claro qué hará y qué no hará la tutoría, ajustar individualmente el estilo de la tutoría, estructurar y planear el proceso desde el comienzo, establecer que la tutoría y el aprendizaje sobre la misma es una

32 SOTO ARANGO, Diana. (2011): Programa Seminario-Taller: Escuela de tutores en el Doctorado Ciencias de la Educación. Tunja, Doctorado Ciencias de la Educación, Universidad Pedagógica y Tecnológica de Colombia, p. 1.

33 PEELO, M. (2011): Understanding supervision and the PhD. London, Continuum International Publishing Group.

${ }^{34}$ SOTO ARANGO, Diana. (2011): Op., cit, p. 1. 
actividad permanente de reflexión, tanto de estudiantes, como de tutores; además socializar y reflexionar sobre las buenas prácticas, propender por su formación continua y cumplir con los estándares e indicadores de evaluación sobre la calidad de la tutoría ${ }^{35}$.

A pesar que las normas institucionales establecen procedimientos para muchas situaciones en las cuales están involucrados los tutores, existen muchas áreas en que no es clara la reglamentación y la decisión queda a cargo de la autonomía individual. Es en estas áreas, en las cuales los tutores experimentan a menudo tensiones ${ }^{36}$, ya que pueden existir argumentos a favor de diferentes cursos de acción y no es claro cuál es el mejor. Es necesario que el tutor conozca de estas posibles tensiones y tome la decisión más acertada, ya que el no hacerlo originará problemas de difícil solución ${ }^{37}$.

La tutoría ha sido objeto de análisis permanente en el Doctorado en Ciencias de la Educación de RUDECOLOMBIA ${ }^{38}$. Dentro de las actividades más recientes se mencionan el Seminario Taller de Autoevaluación y Acreditación de alta calidad ${ }^{39}$, la visita de evaluación de la Asociación Universitaria Iberoamericana de Posgrados al Doctorado de RUDECOLOMBIA ${ }^{40}$ y el Seminario Taller sobre dirección de tesis, realizado durante la reunión número 09 del Comité Curricular Nacional y de Autoevaluación de RUDECOLOMBIA ${ }^{41}$.

${ }^{35}$ ELEY, A. y MURRAY, R. (2009): How to be an effective supervisor. Maidenhead, Mac Graw-Hill

${ }^{36}$ El Diccionario de la Real Academia de la Lengua Española define la tensión como un "estado de oposición u hostilidad latente entre personas o grupos humanos, como naciones, clases, razas, etc". REAL ACADEMIA DE LA LENGUA ESPAÑOLA. (2001): Diccionario de la lengua española. No. 22". España, Real Academia de la Lengua Española, Ed. Madrid.

${ }^{37}$ CRYER, P. (2006): Educational resources for postgraduate research. Manchester. University of Manchester. Descargado el 23 de julio de 2011, de http://www.postgrad_resources.btinternet.co.uk/index.htm

${ }^{38}$ RUDECOLOMBIA, es una red de universidades estatales que se organizaron mediante Convenio el 4 de junio del año 1996. Véase un análisis de la creación y desarrollo de RUDECOLOMBIA en: SOTO ARANGO, Diana. (2004): "El Doctorado Ciencias de la Educación de RUDECOLOMBIA un proyecto de integración universitaria", en: Revista Pensamiento \& Acción. Universidad Pedagógica y Tecnológica de Colombia. Ver: SOTO ARANGO, Diana. (2010): "Redes Universitarias en Colombia. Nueva concepción histórica para la universidad", en: Revista Historia de la Educación Latinoamericana, No. 15, Tunja, Universidad Pedagógica y Tecnológica de Colombia, RUDECOLOMBIA, pp. 241-270.

39 TORRES, M. (2007): "Informe del proceso de autoevaluación. Primer seminario taller internacional de acreditación de alta calidad del Doctorado", en: Boletín Historia de la Educación Latinoamericana. No. 12. Tunja.' Universidad Pedagógica y Tecnológica de Colombia.

${ }^{40}$ ASOCIACIÓN UNIVERSITARIA IBEROAMERICANA DE POSGRADOS (2007): Premio AUIP, programas premiados. 4ta edición. Descargado el 7 de agosto de 2011, de http://www.auip.org/index. php?option $=$ com_content $\&$ view $=$ article\&id $=289 \&$ Itemid $=200 \& l a n g=e s$

${ }^{41}$ COMITÉ CURRICULAR NACIONAL Y DE AUTOEVALUACIÓN DE RUDECOLOMBIA (2010): Acta 09 del 29 y 30 de septiembre de 2010. Bogotá, RUDECOLOMBIA. Archivo RUDECOLOMBIA. 
Durante el Seminario Taller sobre dirección de tesis se identificaron las siguientes tensiones:

\section{Autonomía vs. Dependencia}

Es necesario tener en cuenta que, tanto el tutor, como el estudiante tienen sus propios estilos de tutoría y de aprendizaje. El problema puede surgir cuando no hay compatibilidad entre los dos estilos, por ejemplo, que el tutor sea muy directivo y que el estudiante sea muy autónomo.

Dado que uno de los objetivos del doctorado es lograr la autonomía del estudiante, es necesario discutir con él, el proceso para implementar su independencia, de acuerdo con las expectativas del tutor. El papel de este último es estimular la autonomía como una relación en la cual él asesora, acompaña y estimula el desarrollo del estudiante, en respuesta a las propias ideas y sugerencias del doctorando. El tutor no debe realizar ningún trabajo que le corresponda al estudiante; sin embargo, en ocasiones, cuando el tutor y el estudiante están trabajando en un proyecto común, puede ser difícil diferenciar la contribución hecha por cada uno. En este caso el estudiante debe establecer las fronteras de su trabajo, dirigidos a la realización de la tesis.

La cantidad de trabajo que el tutor debe hacer por los estudiantes, es el que él puede justificar, de acuerdo con su conciencia y la normatividad vigente $^{42}$.

\section{Orientación vs. Coproducción}

Se plantea la disyuntiva si el papel del director debe ser el de orientar y evaluar la producción del estudiante o que sea también un co-productor académico, ya que el director pone en juego su prestigio. Por tal motivo es necesario que se defina desde un comienzo de quién es la idea del trabajo, quién lo desarrolla y quién lo finaliza, con el objeto de establecer la autoría y la co-autoría.

Es necesario aclarar que la propiedad intelectual no es un asunto de generosidad de los tutores o de la buena voluntad de las personas que intervienen en el trabajo. Es aconsejable prever los problemas, revisar las normas y elaborar contratos desde el inicio sobre cómo se manejarán los asuntos relacionados con propiedad intelectual ${ }^{43}$.

${ }^{42}$ CRYER, P. (2006): Óp., cit, p. 5.

${ }^{43}$ CRYER, P. (2006): Óp., cit, p. 5. 
Uno de los problemas surge cuando el estudiante desea publicar como único autor, pero el tutor reclama que él tiene parte en la autoría, ya que aportó con ideas, seguimiento, etc. Por esta razón es necesario establecer los acuerdos acerca de los nombres que aparecerán en la publicación surgida del trabajo. En el caso de que la investigación tenga algún valor financiero, es necesario definir quién tendrá la patente, los derechos de la publicación y cuáles las implicaciones pecuniarias ${ }^{44}$.

\section{Dirección individual vs. Dirección colectiva}

La consideración acerca de esta tensión se presenta, ya que existe la tendencia internacional hacia el establecimiento de la tutoría colectiva ${ }^{45}$, dado que se puede contar con diferentes miradas acerca de la problemática, por ejemplo, a través del grupo de investigación. Los candidatos se exponen a un amplio panorama de perspectivas intelectuales y de experiencia de disciplinas académicas y profesionales, es posible que se especifique el papel de cada uno de los tutores, la responsabilidad del proyecto de investigación recae en varias personas y existe mayor exposición a diferentes estilos de tutoría ${ }^{46}$.

Sin embargo, puede existir desacuerdo entre tutores de diferentes disciplinas acerca del concepto del proyecto de investigación, pueden también surgir dificultades sobre la experiencia de cada uno, el concepto de tutoría que posea, las responsabilidades que tienen y sus estilos propios de tutoría. Otros aspectos a considerar se relacionan con la alta carga de trabajo, la dificultad para poner de acuerdo a más de dos personas para las reuniones y la pérdida de intimidad.

Para prevenir la aparición de problemas, es necesario establecer cuál es el real valor de la co-tutoría y cuáles sus aspectos negativos. El estudiante debe saber cómo manejar la cotutoría. Si se inicia con un solo tutor el trabajo, es necesario establecer en qué momento llamar a un segundo. De igual manera, teniendo en cuenta el tipo de trabajo que realiza el estudiante, se debe definir qué tipo de disciplinas deben tener los cotutores.

${ }^{44}$ CRYER, P. (2006): The research student's guide to success.3ed.Maidenhead, UK, Open University Press.

${ }^{45}$ QUALITY ASSURANCE AGENCY (QAA). (2004): Code of practice for the Assurance of Academic Quality and Standards in Higher Education: Section 1: Posgraduate research, (2nd Ed). Gloucester. Quality Assurance Agency NERAD, M y HEGGELUND, M. (2008): Toward a global PhD: Forces and forms in doctoral education worldwide. Seattle, Washington University Press - POWELL, S. y GREEN, H. (2007): The doctorate worldwide. Maidenhead, MacGraw-Hill.

${ }^{46}$ TAYLOR, S. y BEASLEY, N. (2005): A handbook for doctoral supervisor. Abingdon, UK, Routledge - WELLINGTON, J. (2010): Making supervision work for you. London, Sage Study Skills 
Tutoría metodológica solamente incluyendo lo disciplinar

vs. Tutoría metodológica

Dado que pueden existir planteamientos diferentes acerca del papel del tutor, si éste debe hacer más énfasis en lo metodológico o más en lo disciplinar, es necesario establecer que el objetivo del programa doctoral es la formación integral del estudiante, la cual implica la elaboración del trabajo de investigación en el área del conocimiento, pero que dicho trabajo cumpla con todas las normas establecidas en relación con innovación, originalidad, contribución al conocimiento, etc.

En el Reino Unido, por ejemplo, está establecido, dentro de los procesos de aseguramiento de la calidad, que, aparte de que el tutor tenga competencias metodológicas, es necesario que esté investigando en la disciplina correspondiente al proyecto de investigación del estudiante, con el propósito de asegurar que la dirección y el seguimiento del progreso del estudiante, tenga el soporte de conocimiento actualizado, que apoye el desarrollo de la investigación ${ }^{47}$.

En relación con el tema de la disciplina que trabaja el estudiante, es necesario establecer qué tan fuera de la disciplina que el tutor domina, le puedo permitir trabajar. Lo aconsejable es que el estudiante trabaje dentro de los límites de la disciplina del profesor; si esto no es posible, es necesario nombrar otro tutor que tenga las competencias en el área de trabajo del estudiante o que se aconseje al estudiante la reacomodación del trabajo.

La mayoría de los tutores experimentados sienten que su responsabilidad, en relación con la tesis, es comprobar que se siguen los parámetros establecidos en las normas académicas, tanto para la disciplina respectiva, como la reglamentación institucional sobre los estándares de calidad para la obtención del título ${ }^{48}$.

\section{Tutoría vs. Dirección de tesis}

La tensión se puede presentar, dado que, de acuerdo con el reglamento, el tutor acompaña al estudiante durante el primer $a n ̃ O^{49}$; posteriormente el estudiante puede continuar con el mismo tutor, el cual se denominará director de tesis, o lo puede reemplazar por otra persona.

${ }^{47}$ QUALITY ASSURANCE AGENCY (QAA). (2004): Óp., cit, p. 8.

${ }^{48}$ CRYER, P. (2006): Óp., cit, p. 5.

49 RUDECOLOMBIA (2008): Acuerdo 02 del 2008, por el cual se adopta el Reglamento Estudiantil del Doctorado en Ciencias de la Educación. Cartagena, Doctorado en Ciencias de la Educación RUDECOLOMBIA. 
Se recuerda que el análisis de la reglamentación muestra que en la mayoría del texto se utilizan los dos términos indistintamente ${ }^{50}$, lo cual puede crear confusiones. Aún cuando en el artículo 31 del reglamento estudiantil se definen las funciones del tutor y del director de tesis, estas no proporcionan una diferenciación muy clara entre las dos.

La actividad de formación integral del estudiante durante el desarrollo del programa doctoral tiene varias denominaciones según el país, por ejemplo, el término en el Reino Unido y Australia es "Supervisor", en Estados Unidos de Norteamérica es "Advisor" o "Mentor", en Alemania "Doctor father", en Holanda "Promoter", en España "Director" y en México "Tutor", aunque sus responsabilidades y funciones no varían de manera significativa ${ }^{51}$. Esto demuestra que lo que se debe tener claro son las características, condiciones, funciones y responsabilidades a más del perfil y la experiencia de la o las personas a cargo de dicho proceso, independientemente de la terminología.

Se propone que se estudie la posibilidad de usar solamente una denominación, ya sea tutor o director de tesis, pero que esa persona tenga la responsabilidad de formar integralmente al doctor, en las esferas humanas, sociales, investigativas, éticas, profesionales, físicas e intelectuales.

\section{Tutoría académica solamente vs. Tutoría integral}

Es posible que durante el transcurso del programa, surjan situaciones que afecten el desarrollo del doctorado, que estén relacionadas con aspectos de la vida personal del candidato. Es evidente que, como seres humanos, tanto el tutor, como el estudiante tengan dificultades.

Pueden existir diferencias de opinión, en relación a que el tutor se involucre en aspectos personales del estudiante. Quienes opinan que no, consideran que esta situación puede aumentar su carga de trabajo, que es más aconsejable que sean los consejeros quienes se encarguen de esta tarea. Otros opinan que atender ocasionalmente problemas personales puede enriquecer la relación estudiante tutor, lo cual afecta favorablemente el desempeño del doctorando. En este caso lo importante es definir el momento y la cantidad de ayuda que se puede otorgar, de tal manera que la sesión de tutoría no se convierta en solucionar problemas solamente personales ${ }^{52}$.

50 TORRES, M. (2010): "La tutoría, modelo pedagógico centrado en el estudiante", en: Boletín Historia de la Educación Latinoamericana. No. 14. Tunja, Universidad Pedagógica y Tecnológica de Colombia.

51 EUROPEAN COUNCIL OF DOCTORAL CANDIDATES AND YOUNG RESEARCHERS (EURODOC). (2004): Supervision and training charter for early stage researchers. Brussels, EURODOC. Descargado el 29 de julio de 2011, de http://www.eurodoc.net/temp_policies

52 CRYER, P. (2006): Óp., cit, p. 5. 
Considerando que la formación del doctor es un proceso integral en los aspectos físico, mental, social, ético, profesional e investigativo, es conveniente que aquellos problemas iniciales a nivel personal, que afecten el desarrollo de la investigación, se traten a nivel de la tutoría. El tutor puede ser la primera persona que identifique el problema, ya que en ocasiones el estudiante no lo hace. Si se cree necesario, habrá que remitir al estudiante para una ayuda psicológica, de trabajo social, de bienestar, que puedan apoyar su labor.

Es necesario que el tutor tenga una mentalidad abierta a escuchar al estudiante y a su vez estar pendiente para diagnosticar de manera temprana cualquier dificultad que presente, ya que en ocasiones los mismos estudiantes no aceptan o no son consientes de las dificultades que tienen. Otra posibilidad es que, dentro del equipo de tutores, uno de ellos se encargue de los problemas personales del doctorando ${ }^{53}$.

\section{Autonomía vs. Control normativo de los procesos}

La situación puede surgir cuando, en el desarrollo de la actividad tutorial, surgen situaciones que no están contempladas en las normas y que es necesario que se tome una decisión, con base en el criterio del tutor. Algunos consideran que las reglamentaciones son útiles, especialmente para quienes están iniciando su actividad. Sin embargo, existen algunos tutores quienes piensan que establecer muchas reglamentaciones acerca de la tutoría, les puede quitar la autonomía en el desarrollo de sus actividades ${ }^{54}$.

Se considera necesario, tal como sucede a nivel internacional, que la institución establezca unas guías sobre la actividad tutorial, en las cuales se contemplen aspectos sobre las expectativas que tienen la universidad y los estudiantes hacia el tutor, sus responsabilidades administrativas, investigativas y formativas, la frecuencia de los encuentros con los estudiantes, informes que se deben presentar, establecimiento de programas de desarrollo investigativo, características de los informes de avance, aspectos sobre la entrega de la tesis, duración del doctorado, características de la sustentación ${ }^{55}$ y algunas recomendaciones para solucionar problemas que puedan surgir. Esta reglamentación puede disminuir el porcentaje de reclamos y demandas generados en los vacíos de la normatividad ${ }^{56}$.

53 TAYLOR, S.y BEASLEY, N. (2005): Óp., cit, p. 9.

${ }^{54}$ THE WELLCOME TRUST. (2001): Review of Wellcome Trust PhD Training. The supervisor perspective. London: Wellcome Trust.

${ }^{55}$ WISKER, G. (2005): The good supervisor. Basingstoke, UK, Palgrave Macmillan.

${ }^{56}$ SOTO, D. y TORRES, M. (2010): Relatoría del seminario taller de tutoría y dirección de tesis. Bogotá: Comité Curricular y de Autoevaluación, Doctorado en Ciencias de la Educación de RUDECOLOMBIA 
En algunas ocasiones se aconseja el establecimiento de contratos de aprendizaje, en los cuales se plantean aspectos sobre el tipo de trabajo, de comunicación y de responsabilidades que tendrán las dos partes. Allí se define la cantidad de tiempo y el tipo de soporte que los tutores pueden ofrecer, el comportamiento esperado y las competencias a adquirir ${ }^{57}$.

\section{Otras tensiones}

Las tensiones anteriormente mencionadas son el resultado del análisis hecho por Rudecolombia en el Seminario Taller. La revisión de literatura muestra que existen muchas otras que pueden estar afectando el doctorado, dependiendo de las características propias de cada programa. A continuación se mencionan algunas, como ejemplo, para que sean objeto de análisis por parte de los tutores y se planteen alternativas de solución:

- Originalidad vs. Conformidad

- Formación académica vs. Formación profesional

- Explotación del estudiante por el tutor vs. Aportes para su formación

- Necesidad de la formación del tutor vs. Aumento de burocracia

- Trabajo de tutoría vs. Trabajo administrativo

- Programa basado en cursos vs. Basado en investigación

En resumen, se han analizado las principales tensiones detectadas en el Doctorado en Ciencias de la Educación de RUDECOLOMBIA y algunas alternativas de solución. Es necesario tener en cuenta, dentro del análisis de las tensiones, que existen factores que inciden en el surgimiento y solución de ellas, tales como el número de estudiantes asignados por profesor, las competencias, la experiencia y formación disciplinar, pedagógica e investigativa del tutor, su carga académica dedicada a investigación, administración y docencia, la edad del tutor, la experiencia en publicaciones, la elaboración de artículos, los recursos institucionales y los estilos de aprendizaje de los estudiantes y de enseñanza de los tutores.

\section{Análisis prospectivo de la actividad tutorial en el contexto de la globalización}

Teniendo en cuenta que la actividad tutorial está en el centro de todas las tensiones que pueden generarse en el doctorado, y que la educación doctoral está siendo influenciada por la globalización ${ }^{58}$, es importante tener

${ }^{57}$ WISKER, G. (2005): Óp., cit, p. 13.

${ }^{58}$ Con base en las referencias de Nerad. (2008) y de la Real Academia de la Lengua Española (2001). definimos globalización como un proceso que se relaciona con la interdependencia de los diferentes países del mundo, relacionada con el movimiento intensificado de tecnología, información, ideas, mercados y personas, lo cual produce transformaciones educativas, sociales, económicas y políticas. 
en cuenta cuáles de estos aspectos están afectando el programa y cómo el programa puede afectar la globalización produciendo nuevas generaciones de investigadores y líderes.

Es importante recordar que, dentro de los indicadores de calidad establecidos en los diferentes rankings mundiales, se encuentran los premios y títulos internacionales, la cantidad y calidad de publicaciones en revistas de alta calidad y el factor de impacto relacionado con las citas a los trabajos de investigación realizados. Dentro de las dimensiones de la globalización que afectan la educación doctoral están la mercantilización, referente a que los títulos tienden a ser universalmente equivalentes; la influencia que ejerce la economía en la educación doctoral, el modelo 2 de hacer ciencia, la fuga de cerebros, el uso del inglés como idioma internacional, la tendencia a establecer un modelo común de doctorado, el aseguramiento de la calidad y el tratado de Bolonia ${ }^{59}$.

La combinación de estos factores, junto con el aumento creciente de número de estudiantes de doctorado, haincrementado desproporcionadamente las responsabilidades de tutoría. Esto, acompañado de presiones externas de las entidades financiadoras, las cuales exigen éxito y terminación a tiempo de las investigaciones, al igual que rápida publicación de los resultados en revistas de calidad y el aumento de las presiones en los profesores, en relación con sus responsabilidades docentes y administrativas, pueden ser malas noticias para la calidad de la tutoría. Si los profesores tienen que llevar a cabo sus nuevas y mayores responsabilidades efectivamente, es posible que sea necesario un aumento en los programas de desarrollo de los tutores, mayor soporte para una tutoría exitosa y orientación para prevenir las tensiones, teniendo en cuenta la influencia de los factores de globalización que afectan el programa ${ }^{60}$.

De esta manera se espera que el nuevo programa doctoral tenga, entre otras, las siguientes características: preparación para trabajar en la academia, el gobierno, industria u otra organización; tutoría en equipo, de carácter interdisciplinario; formación en epistemología; trabajo en proyectos colaborativos con otras universidades, centros u organizaciones de investigación; pasantías de formación e investigación internacionales de por lo menos un período académico; vinculación de estudiantes de otros países; establecimiento de programa de desarrollo profesional de los tutores; integración de la educación ética en todos los campos del conocimiento; dominio de otra lengua diferente a la nativa; inclusión de competencias en docencia, liderazgo, comunicación, manejo de proyectos y trabajo en

${ }^{59}$ NERAD, M y HEGGELUND, M. (2008): Óp., cit, p. 9.

${ }^{60}$ ELEY, A. (2001): "Research supervisor training: an irrelevant concept or the key to success?”, en Microbiology Today. Vol. 28. Reading, UK: Society for General Microbiology. 
equipo; proceso de autoevaluación permanente, con miras a acreditación internacional; estímulos a los doctorados colaborativos interinstitucionales e internacionales; doble titulación y formación como ciudadano del mundo ${ }^{61}$.

En resumen, teniendo en cuenta que las tendencias de los doctorados pueden generar tensiones, es necesario realizar un análisis en cada doctorado, sobre qué tensiones potencialmente se pueden presentar, con el objeto de prevenirlas y si ya existen, establecer las causas y valorar las alternativas de solución, para seleccionar la más adecuada. Esto enmarcado en el contexto de la normatividad institucional, relacionada específicamente con la tutoría.

\section{Propuesta de programa de formación tutorial, aplicable al Doctorado en Ciencias de la Educación, RUDECOLOMBIA}

Teniendo en cuenta el análisis presentado sobre la tutoría, sustentado en la comparación y la investigación desarrollada en el Doctorado en Ciencias de la Educación de RUDECOLOMBIA, proponemos unas estrategias para la tutoría en un Doctorado de Educación. Debemos indicar que la tutoría en RUDECOLOMBIA, ha sido permanentemente estudiada, por parte de los Comités Académicos de los Doctorados (CADEs) y el Comité curricular y de autoevaluación ${ }^{62}$ y reglamentada mediante convenios del Consejo de Rectores $^{63}$. Existe también reglamentación acerca de las calidades que debe tener el profesor para poder dirigir actividades de formación doctoral ${ }^{64}$, sin embargo, en Colombia no existen políticas de formación de tutores del doctorado, de ahí que esta propuesta constituye un aspecto innovador.

${ }^{61}$ NERAD, M y HEGGELUNG, M. (2008): Óp., cit, p. 9.

${ }^{62}$ SOTO, Diana. y TORRES, Mario. (2010): Relatoría del seminario taller de tutoría y dirección de tesis. Bogotá, Comité Curricular y de Autoevaluación, Doctorado en Ciencias de la Educación de RUDECOLOMBIA.

${ }^{63}$ Véase un análisis en: SOTO ARANGO, Diana. (2010): "Redes Universitarias en Colombia. Nueva concepción histórica para la universidad”, en: Revista Historia de la Educación Latinoamericana, No. 15, Tunja, Universidad Pedagógica y Tecnológica de Colombia, RUDECOLOMBIA, pp. 241-270. Ver: CONVENIO, 4 junio de 1996. Constitución de la Red. -Convenios de funcionamiento de la red. 17 noviembre del 2008 y 21 noviembre del 2009.- Reglamento General, 28 noviembre 1998; 16 octubre del 2000; 5 agosto del 2003; 16 julio del 2005; -Reglamento Estudiantil, 28 noviembre 1998, 16 de octubre del 2000; 28 de diciembre del 2005; 18 diciembre 2005; 3 de Junio de 2008".

${ }^{64}$ Red de Universidades del Doctorado en Ciencias de la Educación RUDECOLOMBIA. (2008): Acuerdo número 1: "Por el cual se reglamenta el funcionamiento general del doctorado en ciencias de la educación". Cartagena, Colombia: Doctorado en Ciencias de la Educación RUDECOLOMBIA. 
Reiteramos que la propuesta de programa, basada en la reflexión sobre la práctica tutorial ${ }^{65}$, busca establecer mecanismos que apoyen el desarrollo profesional de los tutores ${ }^{66}$ comprometidos en la formación de los doctores ${ }^{67}$; de igual manera plantea estrategias que, por un lado, faciliten el logro de la creatividad, la innovación y el desarrollo continuo y por otro, demuestren a los estudiantes y otras partes interesadas, el profesionalismo que los tutores proporcionan para el apoyo a las experiencias de aprendizaje de los alumnos y la búsqueda de la calidad de dichas experiencias ${ }^{68}$. Para los lineamientos de la propuesta se ha tenido en cuenta la experiencia internacional, especialmente el programa FIRST de Australia, el cual reúne también las experiencias de los países europeos ${ }^{69}$.

Algunos de los logros de aprendizaje a obtener por parte de los tutores, luego de la realización del programa, son los siguientes:

- Autoconciencia de sus concepciones de práctica tutorial.

- Entendimiento de las características de un ambiente de investigación y normatividad institucional.

- Apreciación de los diferentes tipos de buenas prácticas en tutoría.

- Estrategias de reflexión crítica, negociación, entrenamiento y consejería.

- Capacidad de autoevaluación de su eficacia y competencia.

- Habilidades de interacción, comunicación, comprensión y liderazgo

- Pedagogía de la tutoría, políticas de formación en investigación y expectativas de los empleadores y asociaciones estudiantiles.

- Contextualización de la investigación en las problemáticas sociales.

Los contenidos pueden abarcar, entre otros, los siguientes aspectos:

- Concepciones propias sobre investigación y objetivos de la tutoría; experiencias como estudiante y como tutor.

${ }^{65}$ SCHON, Donald. (1998): El profesional reflexivo: Cómo piensan los profesionales cuando actúan. (1 ed). Barcelona, Paidós.

${ }^{66}$ SOTO ARANGO, Diana. (2009): "El profesor universitario de América Latina. Hacia una responsabilidad ética-científico-social", en: Revista Historia de la Educación Latinoamericana, N. 13, Tunja, Universidad Pedagógica y Tecnológica de Colombia. RUDECOLOMBIA, pp. 166-188.

${ }^{67}$ SOTO ARANGO, Diana. (2009): "Los doctorados en Colombia. Un camino hacia la transformación universitaria", en: Revista Historia de la Educación Latinoamericana, $\mathrm{N}^{\circ}$. 12, Tunja, Universidad Pedagógica y Tecnológica de Colombia. RUDECOLOMBIA, pp. 157-195.

${ }^{68}$ THE HIGHER EDUCATION ACADEMY (HEA).(2006): The UK Professional Standards Framework for teaching and supporting learning in bigher education. Heslington, HEA

${ }^{69}$ FOR IMPROVING RESEARCH SUPERVISION AND TRAINING CONSORTIUM (fIRST). (2004): Framework. Melbourne, Australia, FIRST. Descargado el 25 de Julio de 2011, de http://www.first.edu.au/public/first/ framework.html 
- Componentes de un ambiente productivo de aprendizaje de la investigación.

- Etapas básicas y responsabilidades para supervisar un candidato dentro de un límite razonable de tiempo y estrategias y estructuras para acordar el programa de investigación, el estudio del doctorando y la relación tutorial.

- Pedagogía tutorial y habilidades de interacción práctica y comunicación.

- Liderazgo y manejo de los grupos de investigación y del programa de investigación del doctorado.

Las estrategias de aprendizaje se basan en seminarios teóricos, talleres, oportunidades para el aprendizaje práctico y reflexivo, mediante el encuentro de académicos nuevos con los de más experiencia, en los cuales se hagan diagnósticos de las prácticas tutoriales, estudios de casos e incidentes críticos. De igual manera se plantea el acceso electrónico a la literatura y a grupos en línea.

Se sugiere que esta propuesta se discuta inicialmente en los CADEs, junto con toda la comunidad educativa; allí se debe analizar la experiencia obtenida sobre la tutoría y dirección de tesis, en los 15 años de existencia del doctorado, actualizar la información disponible a nivel mundial y adecuar la propuesta, de acuerdo con las necesidades locales, regionales y nacionales. Luego se definirá la aceptación y desarrollo de la propuesta, por parte de los entes administrativos pertinentes.

Se deben tener en cuenta aspectos como alcance, cobertura, temas, tipo de evento, recursos, responsabilidad, participantes, lugar y momento de realización, tipo de evaluación, presupuesto, certificación y seguimiento.

En resumen, se presenta la propuesta de programa de formación del tutor, de la cual hacen parte la contextualización, los logros de aprendizaje, los contenidos, las estrategias de aprendizaje y algunas recomendaciones para su implementación. Se aclara que esta es una fase inicial de la propuesta, la cual, luego de la socialización de las experiencias de los 15 años del funcionamiento del doctorado y su confrontación con la experiencia mundial, puede hacer parte de las políticas de desarrollo profesional de los tutores. 


\section{CONCLUSIÓN}

Algunas de las problemáticas generales de los doctorados, comunes en la mayoría de países, se refieren a prolongados tiempos de terminación del programa, bajas tasas de finalización, poca relación con el sector productivo, falta de preparación para el empleo y tendencia de los programas de graduar doctores para que trabajen solo en universidades.

Se encontró que dichos problemas generales de los doctorados, están relacionados con la actividad tutorial, lo que demuestra que gran parte de la responsabilidad del programa recae en el desempeño efectivo del tutor, y que, por otro lado, la mejoría en la calidad de las funciones del tutor incide en la solución de los problemas del doctorado.

Se identificó que las tensiones existentes en el desarrollo de la actividad tutorial del Doctorado en Ciencias de la Educación de Rudecolombia, son comunes en varios programas de doctorado a nivel internacional. Estas tensiones se relacionan con la autonomía, la producción académica, la tutoría en equipo, las responsabilidades mutuas, la formación en investigación, la denominación del proceso, los componentes de la formación, la relación con el proceso curricular, la evaluación de los estudiantes y la normatividad. Se plantearon alternativas de solución para algunas de las tensiones, las cuales permitirán que los tutores desempeñen mejor su labor y, al mismo tiempo, constituyan un aporte para establecer la normatividad. Estas tensiones fueron contextualizadas desde la perspectiva de la globalización y las tendencias mundiales de la formación doctoral.

Se recomienda que se evalúe la actividad tutorial y, de acuerdo con el diagnóstico elaborado, se adecúe la propuesta de programa de formación y/o actualización de los tutores y directores de tesis. Dicho programa puede aplicarse al Doctorado en Ciencias de la Educación de RUDECOLOMBIA, el cual debe ser valorado por la comunidad académica y los entes administrativos, para su recomendación final y puesta en marcha.

Dentro de las recomendaciones, es necesario establecer una línea de investigación en el grupo HISULA, dedicada evaluar las necesidades de estudiantes y tutores sobre la actividad tutorial, monitorizar el impacto de dicha tutoría y establecer planes de mejoramiento, que hagan parte de los proyectos de desarrollo estudiantil y profesoral. Se propone que, dentro del proceso de autoevaluación y acreditación, se incremente el interés que la tutoría debe tener como una de las características de dicho proceso. Se recomienda revisar y actualizar los reglamentos general y estudiantil, para tener en cuenta los hallazgos del estudio y la propuesta de programa de formación. 


\section{FUENTES}

\section{Legislación RUDECOLOMBIA}

Actas consejo de rectores de RUDECOLOMBIA (1996 - 2011).

Actas comite curricular de RUDECOLOMBIA (1996 - 2011).

CONVENIO, 4 junio de 1996. Constitución de la Red.

CONVENIOS DE FUNCIONAMIENTO DE LA RED. 17 noviembre del 2008 y 21 noviembre del 2009.

CONVENIOS DE FUNCIONAMIENTO DE LA RED. 17 noviembre del 2008 y 21 noviembre del 2009.

REGLAMENTO GENERAL, 28 noviembre 1998; 16 octubre del 2000; 5 agosto del 2003; 16 julio del 2005.

REGLAMENTO ESTUDIANTIL, 28 noviembre 1998, 16 de octubre del 2000; 28 de diciembre del 2005; 18 diciembre 2005. 3 de Junio de 2008.

COMITÉ CURRICULAR NACIONAL Y DE AUTOEVALUACIÓN DE RUDECOLOMBIA (2010) Acta 09 del 29 y 30 de septiembre de 2010. Bogotá, RUDECOLOMBIA.

COUNCIL OF AUSTRALIAN DEANS AND DIRECTORS OF GRADUATE STUDIES. (1999): Statement on Skills Development for Research Students. Townsville, Australia, DDOGS. Descargado el 23 de julio de 2011, de http:// www.ddogs.edu.au/files?folder_id=2123770849.

COUNCIL OF AUSTRALIAN DEANS AND DIRECTORS OF GRADUATE STUDIES. (2008): Framework for Best Practice in Doctoral Research Education in Australia. Townsville, Australia, DDOGS. Descargado el 23 de julio de 2011, de http://www.ddogs.edu.au/files?folder_id=2123770849.

COUNCIL OF GRADUATE SCHOOLS. (2004): Completion Project. Washington, CGS. Descargado el 23 de julio de 2011, de http://www.phdcompletion.org/ promising/mentoring.asp.

FOR IMPROVING RESEARCH SUPERVISION AND TRAINING CONSORTIUM (fIRST). (2004): How FIRST Works. Melbourne, Australia, FIRST. Descargado el 25 de Julio de 2011, de http:/ / www.first.edu.au/index.html

QUALITY ASSURANCE AGENCY (QAA). (2004): Code of practice for the Assurance of Academic Quality and Standards in Higher Education: Section 1: Posgraduate research, (2nd Ed). Gloucester, Quality Assurance Agency.

RED DE UNIVERSIDADES DEL DOCTORADO EN CIENCIAS DE LA EDUCACIÓN RUDECOLOMBIA (2008): "Acuerdo número 1: Por el cual se reglamenta el funcionamiento general del doctorado en ciencias de la educación”. Cartagena, Colombia: Doctorado en Ciencias de la Educación RUDECOLOMBIA.

RUDECOLOMBIA (2008): "Acuerdo 02 del 2008, por el cual se adopta el Reglamento Estudiantil del Doctorado en Ciencias de la Educación”. Cartagena, Doctorado en Ciencias de la Educación RUDECOLOMBIA. 


\section{REFERENCIAS}

ALCÁNTARA, Armando; MALO, Salvador y FORTES, Mauricio. (2008): Doctoral Education in África, South America, and México. México, en Toward a global PhD: Forces and forms in doctoral education worldwide. NERAD, Maresi y HEGGELUND, Mimi. Seattle. Washington University Press.

ASOCIACIÓN UNIVERSITARIA IBEROAMERICANA DE POSGRADOS. (2007): Premio AUIP, programas premiados. 4ta. edición. Descargado el 7 de agosto de 2011, de http:/ / www.auip.org/index.php?option=com_content \&view $=$ article\&id $=289 \&$ Itemid $=200 \&$ lang $=$ es.

CLEMENTS, Margaret y ALCÁNTARA Armando. (2005): "Mentoring practices in doctoral program in Mexico and the United States: Growing Wiser Together", paper prepared for the $49^{\text {th }}$ Annual Meeting of the comparative and international Education Society, 22-26 March. Palo Alto, CA, Stanford University.

CRYER, Pat. (2006): "Educational resources for postgraduate research". Manchester, University of Manchester. Descargado el 23 de julio de 2011, de http:/ / www.postgrad_resources.btinternet.co.uk/index.htm.

CRYER, P. (2006): The research student's guide to success. 3 ed. Maidenhead, UK, Open University Press.

DEPARTMENT OF EDUCATION, TRAINING AND YOUTH AFFAIRS, AUSTRALIA. (2001): "Factors Associated with completion of research higher degrees", en: Higher education series Report. No. 37. Sydney, Higher Education Division.

EUROPEAN COUNCIL OF DOCTORAL CANDIDATES AND YOUNG RESEARCHERS (EURODOC). (2004): "Supervision and training charter for early stage researchers. Brussels”, EURODOC. Descargado el 29 de julio de 2011, de http://www.eurodoc.net/temp_policies

ELEY, A. (2001): "Research supervisor training: an irrelevant concept or the key to success?”, en: Microbiology Today. Vol. 28. Reading, UK: Society for General Microbiology.

ELEY, A. y MURRAY, R. (2009): How to be an effective supervisor. Maidenhead, MacGraw-Hill.

EUROPEAN COUNCIL OF DOCTORAL CANDIDATES AND YOUNG RESEARCHERS (EURODOC). (2004): "Supervision and training charter for early stage researchers”. Brussels, EURODOC. Descargado el 29 de julio de 2011, de http://www.eurodoc.net/temp_policies.

GREEN, Howard. (2008): "Doctoral Education in Europe. United Kingdom", en: Toward a global PhD: Forces and forms in doctoral education worldwide. NERAD, Maresi y HEGGELUND, Mimi. Seattle. Washington University Press. 
KEHM, Bárbara. (2007): Doctoral Education in Germany: between tradition and reform, en The doctorate worldwide. POWELL Stuart y GREEN Howard. Maidenhead. McGraw-Hill.

KEHM, Bárbara. (2008): "Doctoral Education in Europe. Germany", en: Toward a global PhD: Forces and forms in doctoral education worldwide". NERAD, Maresi y HEGGELUND, Mimi. Seattle. Washington University Press.

KEMP, David. (1999): Knowledge and innovation: a policy statement and research training. Camberre, Departament of Education, Training and Youth Affairs.

MALO, Salvador. (2007): "Doctoral education in México", en: The doctorate worldwide. POWELL, Stuart y GREEN, Howard. Maidenhead. MacGrawHill.

MENTORING OTHERS RESULTS IN EXCELLENCE (MORE). (2011): "Graduate student mentoring at the University of Michigan". Michigan, University of Michigan. Descargado el 29 de julio de 2011, de http://www. more.umich.edu/mentoring/graduate-student-mentoring/.

MORENO, María. (2007): "Experiencias de formación y formadores en programas de doctorado en educación", en: Revista Mexicana de Investigación Educativa, Vol. 12, No. 33. Guadalajara, Universidad de Guadalajara.

NERAD Maresi. (2007): "Doctoral education in USA", en: The doctorate worldwide. POWELL, Stuart y GREEN, Howard. Maidenhead. MacGrawHill.

NERAD, Maresi. (2008): "Doctoral Education in North America. United States of America", en: Toward a global PhD: Forces and forms in doctoral education worldwide. NERAD, Maresi. y HEGGELUND, Mimi. Seattle. Washington University Press.

NERAD, M y HEGGELUND, M. (2008): "Toward a global PhD: Forces and forms in doctoral education worldwide”. Seattle, Washington University Press.

SOTO ARANGO, Diana. (2004): "El Doctorado Ciencias de la Educación de RUDECOLOMBIA un proyecto de integración universitaria", en: Revista Pensamiento E Acción. Universidad Pedagógica y Tecnológica de Colombia.

SOTO ARANGO, Diana. (2009): "El profesor universitario de América Latina. Hacia una responsabilidad ética-científico-social", en: Revista Historia de la Educación Latinoamericana, No. 13, Tunja, Universidad Pedagógica y Tecnológica de Colombia. RUDECOLOMBIA.

SOTO ARANGO, Diana. (2009): "Los doctorados en Colombia. Un camino hacia la transformación universitaria", en: Revista Historia de la Educación Latinoamericana, No. 12, Tunja, Universidad Pedagógica y Tecnológica de Colombia. RUDECOLOMBIA.

SOTO ARANGO, Diana. (2010): "Redes Universitarias en Colombia. Nueva concepción histórica para la universidad”, en: Revista Historia de la Educación 
Latinoamericana, No. 15, Tunja, Universidad Pedagógica y Tecnológica de Colombia. RUDECOLOMBIA.

SOTO ARANGO, Diana. (2012): Programa Seminario-Taller: Escuela de tutores en el Doctorado en Ciencias de la Educación. Tunja, Doctorado en Ciencias de la Educación, Universidad Pedagógica y Tecnológica de Colombia.

SOTO, Diana y TORRES, Mario. (2010): Relatoría del seminario taller de tutoría y dirección de tesis. Bogotá, Comité Curricular y de Autoevaluación, Doctorado en Ciencias de la Educación de RUDECOLOMBIA.

PEELO, M. (2011): Understanding supervision and the PhD. London, Continuum International Publishing Group.

POWELL, S. y GREEN, H. (2007): The doctorate worldwide. Maidenhead, MacGraw-Hill.

REAL ACADEMIA DE LA LENGUA ESPAÑOLA. (2001): "Diccionario de la lengua española”. 22 ed. Madrid, España, Real Academia de la Lengua Española.

RIBEIRO, Renato. (2007): "Doctoral education in Brazil", en: The doctorate worldwide. POWELL, Stuart. y GREEN, Howard. Maidenhead. MacGrawHill.

RIBEIRO, Renato. (2008): "Doctoral Education in África, South América, México, Brazil", en: Toward a global PhD: Forces and forms in doctoral education worldwide. NERAD, Maresi y HEGGELUND, Mimi. Seattle. Washington University Press.

SCHON, Donald. (1998): El profesional reflexivo: Cómo piensan los profesionales cuando actúan 1 ed. Barcelona, Paidós.

TAYLOR, S.y BEASLEY, N. (2005): A bandbook for doctoral supervisor. Abingdon, UK, Routledge.

THE HIGHER EDUCAITON ACADEMY (HEA). (2005): "First accredited programme for training in supervising postgrads". Heslington, HEA. Descargado el 24 de julio de 2011, de: http://www.heacademy.ac.uk/news/ detail/accredited_programme_training_supervising_postgrads.

THE WELLCOME TRUST. (2001): Review of Wellcome Trust PhD Training. The supervisor perspective. London: Wellcome Trust.

TORRES, M. (2007): "Informe del proceso de autoevaluación. Primer seminario taller internacional de acreditación de alta calidad del Doctorado", en: Boletín Historia de la Educación Latinoamericana. No. 12. Tunja, Universidad Pedagógica y Tecnológica de Colombia.

TORRES, M. (2010): "La tutoría, modelo pedagógico centrado en el estudiante”, en: Boletín Historia de la Educación Latinoamericana. No. 14. Tunja, Universidad Pedagógica y Tecnológica de Colombia.

URQUHART, Kirstie. (2001): “Superior supervisión”, en: Science Career Magazine. Nueva York, American Association for the Advancement of 
Science. Descargado el 23 de julio de 2011, De: http://sciencecareers. sciencemag.org/career_magazine/previous_issues/articles/2001_11_02/ noDOI.5857770442054790723.

VITAE. (s.f.). Supervisors $\mathcal{E}$ managers. Cambridge, Careers Research and Advisory Centre (CRAC): Descargado el 25 de julio de 2011, de http://www.vitae. ac.uk/policy-practice/1389/Supervisors--managers.html.

WELLINGTON, J. (2010): Making supervision work for you. London, Sage Study Skills.

WISKER, G. (2005): The good supervisor. Basingstoke, UK, Palgrave Macmillan.

TORRES CALIXTO, Mario Gabriel. (2011): "La tutoría en programas de doctorado. Tensiones tutoriales doctorado en ciencias de la educación RUDECOLOMBIA" en: Revista Historia de la Educación Latinoamericana Vol. 13 No. 17, Tunja, Universidad Pedagógica y Tecnológica de Colombia, RUDECOLOMBIA, SHELA- HISULA. pp. 315-344 